SESSION V : Innovation en génie civil - génie côtier

\title{
Reconstitution de sols argileux en laboratoire
}

\author{
A. Benamar \\ Maitre de conférences, Université du Havre, Laboratoire de Mécanique.
}

\section{Résumé}

La validité des paramètres d'état des sols mesurés au laboratoire est conditionnée en partie par le mode de prélèvement des carottes de sol. Afin de pallier cet inconvénient, des échantillons d'argile sont reconstitués en laboratoire en examinant la reproductibilité et l'homogénéité des éprouvettes obtenues. Cet article traite d'une expérience de reconstitution d'argile dans un moule œdométrique spécifique de grandes dimensions. Les résultats obtenus montrent que la procédure de reconstitution des échantillons utilisant un chargement œdométrique adapté s'avère intéressante. Les caractéristiques mécaniques mesurées lors des essais effectués sur des éprouvettes reconstituées dans les mêmes conditions, sont très peu variables.

\section{Introduction}

Le prélèvement d'échantillons de sols sur site pose des problèmes certains quant à l'homogénéité des éprouvettes, le remaniement du sol et la reproductibilité de l'opération. Les caractéristiques mécaniques mesurées sur de telles éprouvettes peuvent varier de façon importante sans pouvoir situer la cause. Cette difficulté de ramener du site des échantillons intacts est d'autant plus grande que le sol est moins dense comme c'est le cas des sols vaseux.

Pour faire varier les procédures d'essai et étudier leur influence sur les paramètres mesurés au laboratoire, il est important de disposer d'éprouvettes identiques ou, au moins, présentant un faible taux de dispersion sur les propriétés physiques et mécaniques du sol. La validité des paramètres d'état des sols mesurés au laboratoire est conditionnée en partie par le mode de prélèvement des échantillons. Le remaniement est le résultat de l'ensemble des opérations depuis l'extraction de l'éprouvette de son milieu naturel jusqu'au moment où elle est soumis à la procédure d'essai pour laquelle elle a été prélevée. Les échantillons sont souvent extraits d'un sol affecté par l'opération de forage qui précède le carottage.

La reconstitution d'échantillons de sol au laboratoire à partir de la matrice solide, réduite à une poudre, s'avère comme une alternative intéressante permettant de s'affranchir des aléas de la procédure traditionnelle de prélèvement sur le terrain. L'opération doit être conduite en se souciant à chaque instant de la 
reproductibilité et de l'homogénéité des éprouvettes de sol. Différents laboratoires ont utilisé des méthodes de consolidation d'échantillons de sols naturels ou reconstitués. Les modes de confection des éprouvettes consistent soit à réaliser des massifs de sol dans de gros cdomètres dans lesquels les éprouvettes seront carottées, soit à confectionner des échantillons dans des moules aux dimensions appropriées. Lorsqu'il s'agit des essais en modèle réduit, notamment en centrifugeuse, des massifs de sol à l'échelle sont reconstitués avec soin.

Dans le cadre d'une expérience ayant pour objet de simuler au laboratoire le battage d'un pieu à travers une couche homogène de sol cohérent, des éprouvettes d'argile (kaolin) ont été réalisées sur place autour d'une barre d'acier. L'opération de reconstitution exécutée dans un moule œdométrique a nécessité un dispositif de drainage adapté.

\section{Le dispositif adométriaue}

\subsection{La cellule}

Le moule œdométrique ( figure 1) est haut de $900 \mathrm{~mm}$ et formé de deux demi-coquilles de diamètre intérieur $200 \mathrm{~mm}$. Il est surmonté d'un piston étanche mu par un vérin creux, permettant d'appliquer une charge verticale à l'échantillon pendant la consolidation. Le vérin hydraulique est suspendu et peut coulisser le long de la barre. Il a une course de $250 \mathrm{~mm}$, une pression de service de $20 \mathrm{MPa}$ correspondant à une pression de 1.75 MPa sur l'échantillon.

Pendant la consolidation le drainage est assuré par deux disques poreux aux extrémités de l'échantillon. L'eau est récupérée par deux orifices dans les disques poreux. Un géotextile de type non tissé aiguilleté est disposé latéralement autour de l'échantillon pour améliorer le drainage. Le piston étanche est mû par le vérin le long du moule sans endommager le géotextile mis en place.

\subsection{L'échantillon}

L'échantillon est une argile reconstituée au laboratoire à partir de $25 \mathrm{~kg}$ de poudre de Kaolin provenant de Bretagne (Ploërmel). La poudre et l'eau sont mélangés sous vide dans un malaxeur tournant à vitesse constante, à une teneur en eau voisine de $92 \%$ (de l'ordre du double de la limite de liquidité). Le mélange ainsi constitué est saturé, et se présente dans un état de consistance qui permet sa mise en place facile dans le moule. En plus du dispositif de drainage aux extrémités de l'échantillon, l'eau peut être évacuée à l'interface barre - argile lorsque les presse-étoupes (éléments coniques en caoutchouc), qui servent de dispositif d'étanchéité autour de la barre, sont desserés (figure 1).

\subsection{La consolidation}

Après sa mise en place, l'argile est consolidée à l'aide du vérin qui applique à l'échantillon une pression constante. Le chargement s'effectue par 
paliers: le premier chargement par le verin (après le poids du piston qui applique une pression de $0.60 \mathrm{kPa}$ ) correspond à une pression $10 \mathrm{kPa}$ sur l'échantillon. Les incréments de charge ont été choisis selon le mode opératoire de l'essai odométrique : les pressions sont doublées à chaque incrément. On ne passe d'un palier de chargement au palier suivant que lorsque le tassement correspondant au premier palier est stabilisé (pression interstitielle nulle). Ce critère est vérifié par un capteur de pression interstitielle placé dans l'échantillon (figure 1). La durée d'un palier de chargement varie de 24 heures à 72 heures selon la dissipation des pressions interstitielles. En effet, la compressibilité de l'argile dépend beaucoup de la vitesse de chargement. La consolidation dure environ deux semaines et réduit les dimensions de l'échantillon à $0.52 \mathrm{~m}$ de long et $0.20 \mathrm{~m}$ de diamètre. La pression finale atteinte est de $280 \mathrm{kPa}$ à cause de la limite de la course du verin. La consolidation est poursuivie au besoin dans une cellule triaxiale.

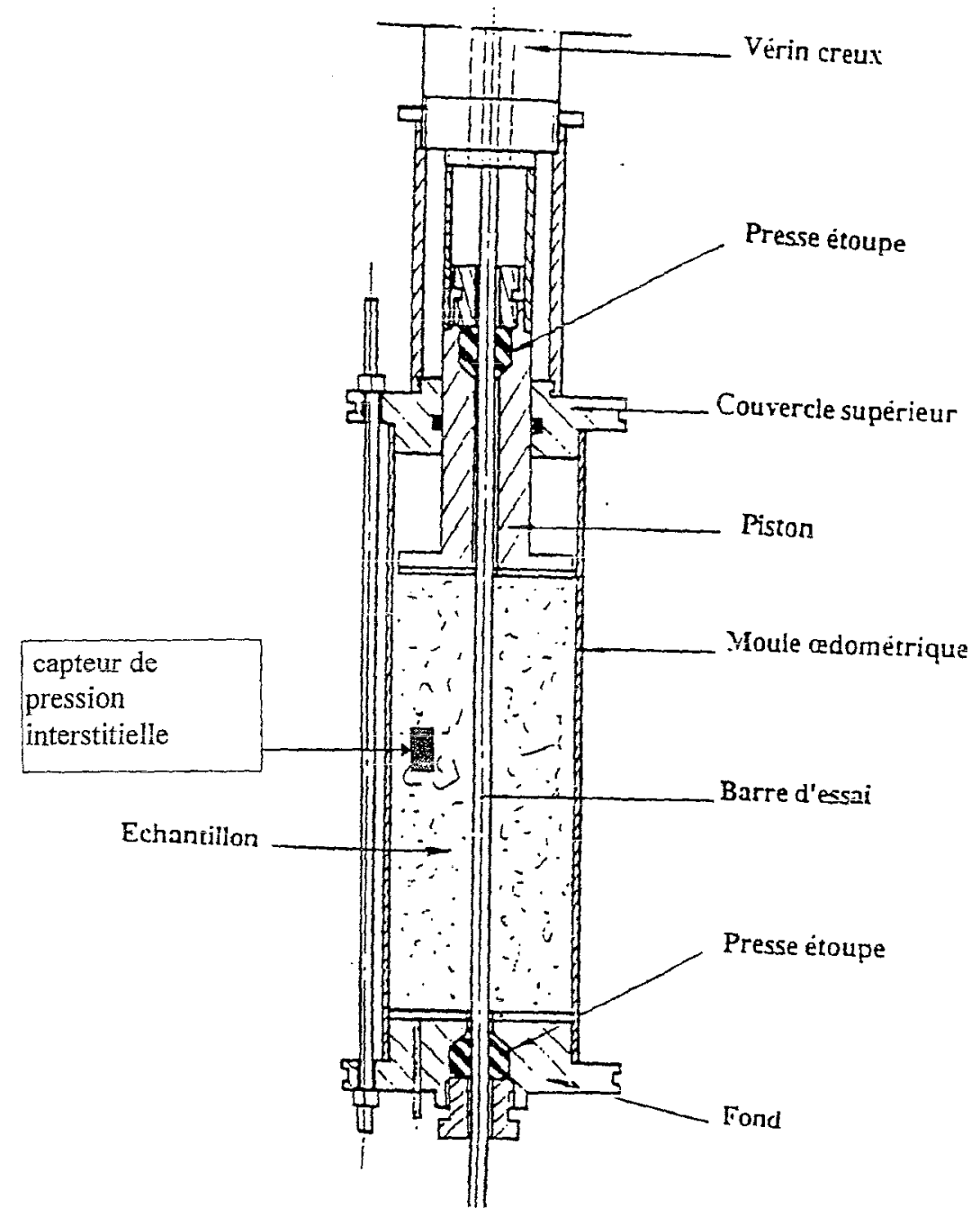

Figure 1: Coupe schématique du moule œdométrique: $(\varnothing 200, \mathrm{H}=900)$. 
A la fin de la consolidation œdométrique l'indice des vides et le poids volumique sont obtenus par les relations:

$$
\begin{aligned}
& e=\frac{\gamma_{s}}{\gamma_{w}} \\
& \gamma=\frac{\gamma_{s}+e}{1+e} \gamma_{w}
\end{aligned}
$$

où $\gamma_{s}$ est le poids volumique des grains solides, et $\gamma_{W}$ le poids volumique de l'eau.

La consolidation réduit l'échantillon en hauteur de 35\%, faisant varier l'indice des vides de 2.41 à 1.04 . Le poids volumique final des échantillons obtenus est de $18 \mathrm{kN} / \mathrm{m}^{3}$ environ avec une teneur en eau voisine de $40 \%$ pour une contrainte de consolidation de $280 \mathrm{kPa}$. L'indice de plasticité et la cohésion non drainée sont respectivement de 23 et $65 \mathrm{kPa}$. La courbe de consolidation de la figure 2 montre que la vitesse de déformation de l'échantillon est quasi-constante au cours de l'essai de reconstitution. La courbe œdométrique d'un échantillon représentée sur la figure 3 permet de déduire l'indice compression $\mathrm{C}_{\mathrm{c}}$ du sol.

Les pressions de consolidation appliquées à l'échantillon sont suffisamment importantes pour vaincre les frottements du piston avec les parois. de plus, le piston comporte un anneau en caoutchouc qui permet de réduire ces frottements.

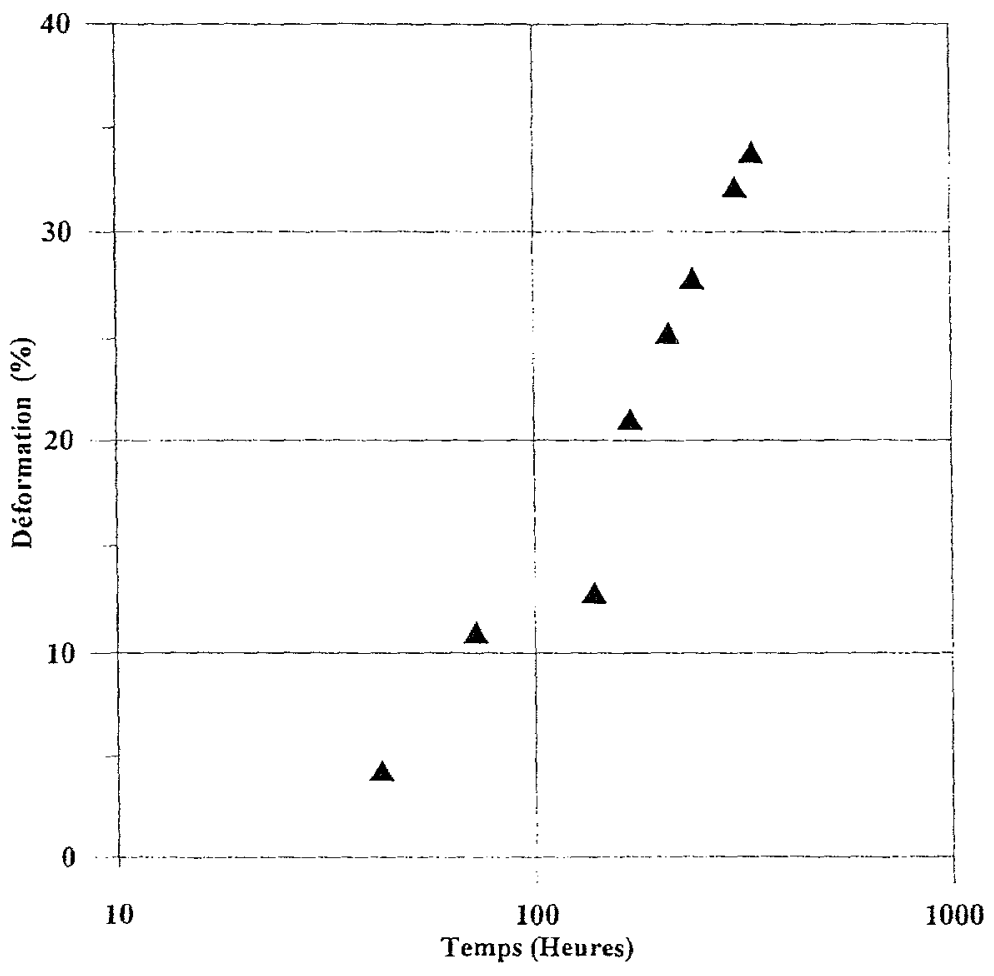

Figure 2: Courbe de consolidation 


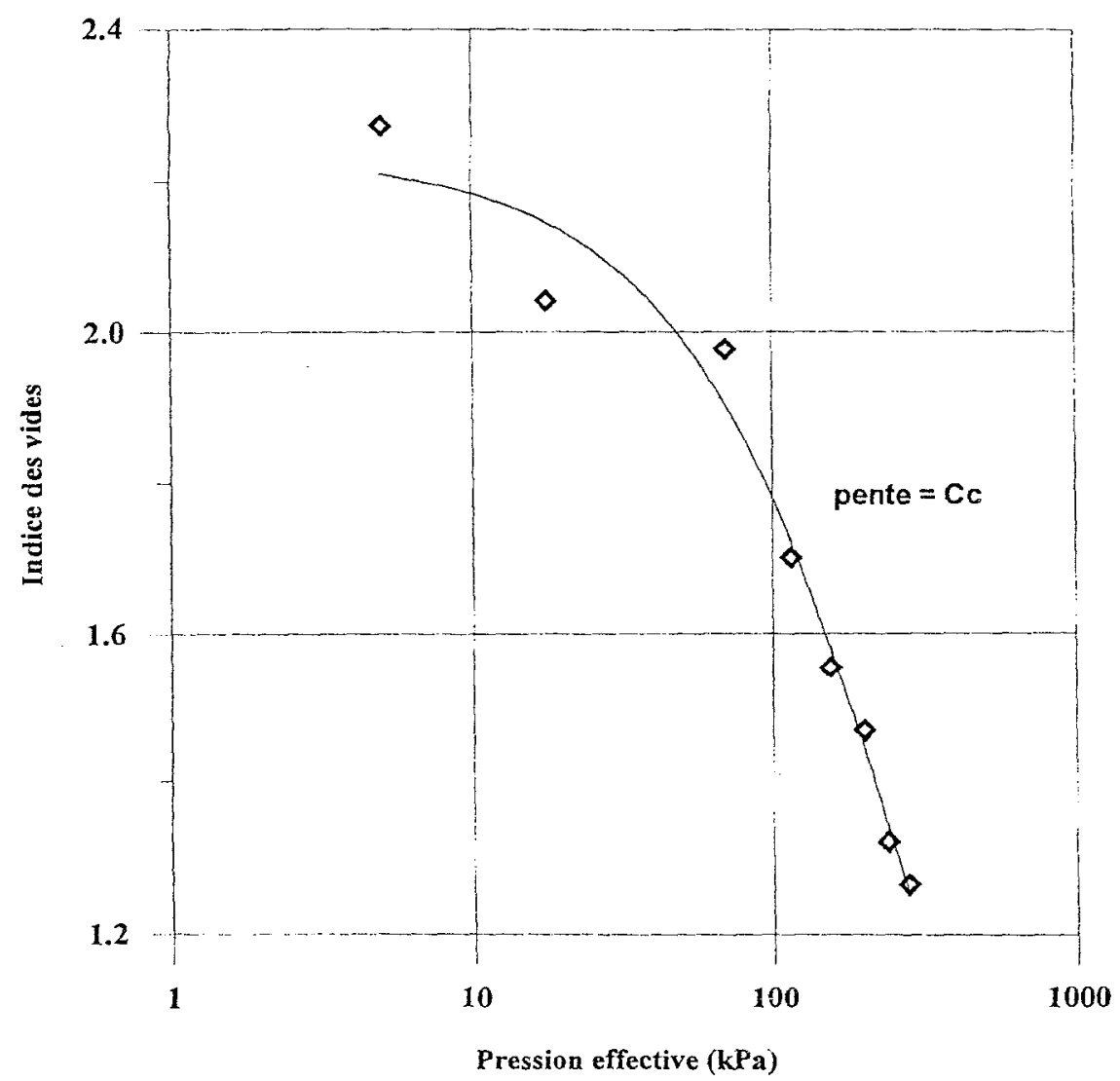

Figure 3 : Courbe cedométrique

A la fin de la consolidation, et avant d'effectuer les essais de battage, certains paramètres physiques des échantillons sont mesurés. Le tableau I ci-après résume ces paramètres ainsi que la caractéristique de compressibilité. L'indice decompression étant la pente de la partie linéaire de la courbe œdométrique de la figure 3 .

\begin{tabular}{|l|c|c|c|c|c|c|c|c|c|}
\hline Echantillon & 1 & 2 & 3 & 4 & 5 & 6 & 7 & 8 & 9 \\
\hline $\begin{array}{l}\text { Teneur en eau } \\
\text { initiale } \mathrm{W}_{\mathrm{i}}(\%)\end{array}$ & 88.5 & 90.0 & 88.0 & 88.0 & 80.8 & 93.0 & 88.0 & 100.0 & 92.1 \\
\hline $\begin{array}{l}\text { Pression de conso } \\
\text { lidation }(\mathrm{kPa})\end{array}$ & 280 & 280 & 280 & 280 & 280 & 255 & 152 & 240 & 280 \\
\hline $\begin{array}{l}\text { Indice des vides } \\
\text { initial } \mathrm{e}_{0}\end{array}$ & 2.31 & 2.36 & 2.30 & 2.30 & 2.11 & 2.43 & 2.30 & 2.62 & 2.41 \\
\hline $\begin{array}{l}\text { Indice de } \\
\text { compression } \mathrm{C}_{c}\end{array}$ & 0.20 & 0.21 & 0.23 & 0.30 & 0.25 & 0.19 & 0.21 & 0.29 & 0.24 \\
\hline
\end{tabular}

Tableau I: Caractéristiques physiques des échantillons en consolidation . 


\section{Caractérisation des éprouvettes reconstituées}

Pour les besoins des essais de battage, certains échantillons finissent la consolidation dans une cellule triaxiale sous une pression pouvant atteindre 500 $\mathrm{kPa}$. A la fin des essais différents essais de laboratoire sont effectués pour caractériser l'argile reconstituée. Le tableau II ci-après récapitule les valeurs de la teneur en eau $W$ et de la cohésion non drainée $C_{u}$ mesurées le long de l'échantillon. Les teneurs en eau indiquées correspondent à des sections différentes de l'échantillon. Les niveaux 1 à 3 correspondent successivement à la face supérieure, le milieu et la face inférieure de l'éprouvette de sol. La cohésion non drainée désigne une valeur moyenne (sur les 3 valeurs des différents niveaux) mesurée au scissomètre de poche. Les teneurs en eau sont mesurées avec une précision de l'ordre de $5 \%$ (erreur sur la mesure du poids) et la précision sur la cohésion non drainée peut être estimée à $10 \%$.

\begin{tabular}{|l|c|c|c|c|c|c|c|c|c|}
\hline Echantillon & 1 & 2 & 3 & 4 & 5 & 6 & 7 & 8 & 9 \\
\hline $\begin{array}{l}\text { Pression } \\
\text { finale (kPa) }\end{array}$ & 500 & 500 & 500 & 500 & 500 & 400 & 200 & 500 & 300 \\
\hline $\begin{array}{l}\text { W (\%) } \\
\text { au niveau 1 }\end{array}$ & 33 & 32 & 33 & 34.5 & 34.5 & 36.3 & 36 & 34 & 36.8 \\
\hline $\begin{array}{l}\text { W }(\%) \\
\text { au niveau 2 }\end{array}$ & 34 & 33.4 & 33.7 & 34.7 & 33.8 & 35.6 & 38 & 33.6 & 36.6 \\
\hline $\begin{array}{l}\text { W }(\%) \\
\text { au niveau 3 }\end{array}$ & 35 & 34.4 & 34 & 35.3 & 34.2 & 36.2 & 40 & 32 & 36 \\
\hline $\begin{array}{l}\text { Cohésion non } \\
\text { drainée }(\mathrm{kPa})\end{array}$ & 68 & 70 & 69 & 73 & 71 & 52 & 36 & 79 & 50 \\
\hline
\end{tabular}

Tableau II: Caractéristiques physiques et mécaniques des échantillons reconstitués

Les résultats ci-dessus montrent d'une part une homogénéité des propriétés physiques et mécaniques de l'argile dans un même échantillon, et d'autre part l'homogénéité d'un échantillon à l'autre quand les conditions de consolidation sont identiques. A la même pression de consolidation ( $500 \mathrm{kpa}$ ), la valeur moyenne de la teneur en eau dans toutes les éprouvettes est de $34 \%$ avec un écart-type voisin de 0.7. La cohésion non drainée moyenne est $70 \mathrm{kPa}$ pour un écart-type de 2.1 .

La teneur en eau moyenne du sol reconstitué et sa cohésion non drainée moyenne varient avec la pression de consolidation. Cette variation est illustrée sur la figure 4 et montre que les caractéristiques mécaniques du sol peuvent être contrôlées par les conditions de consolidation. 


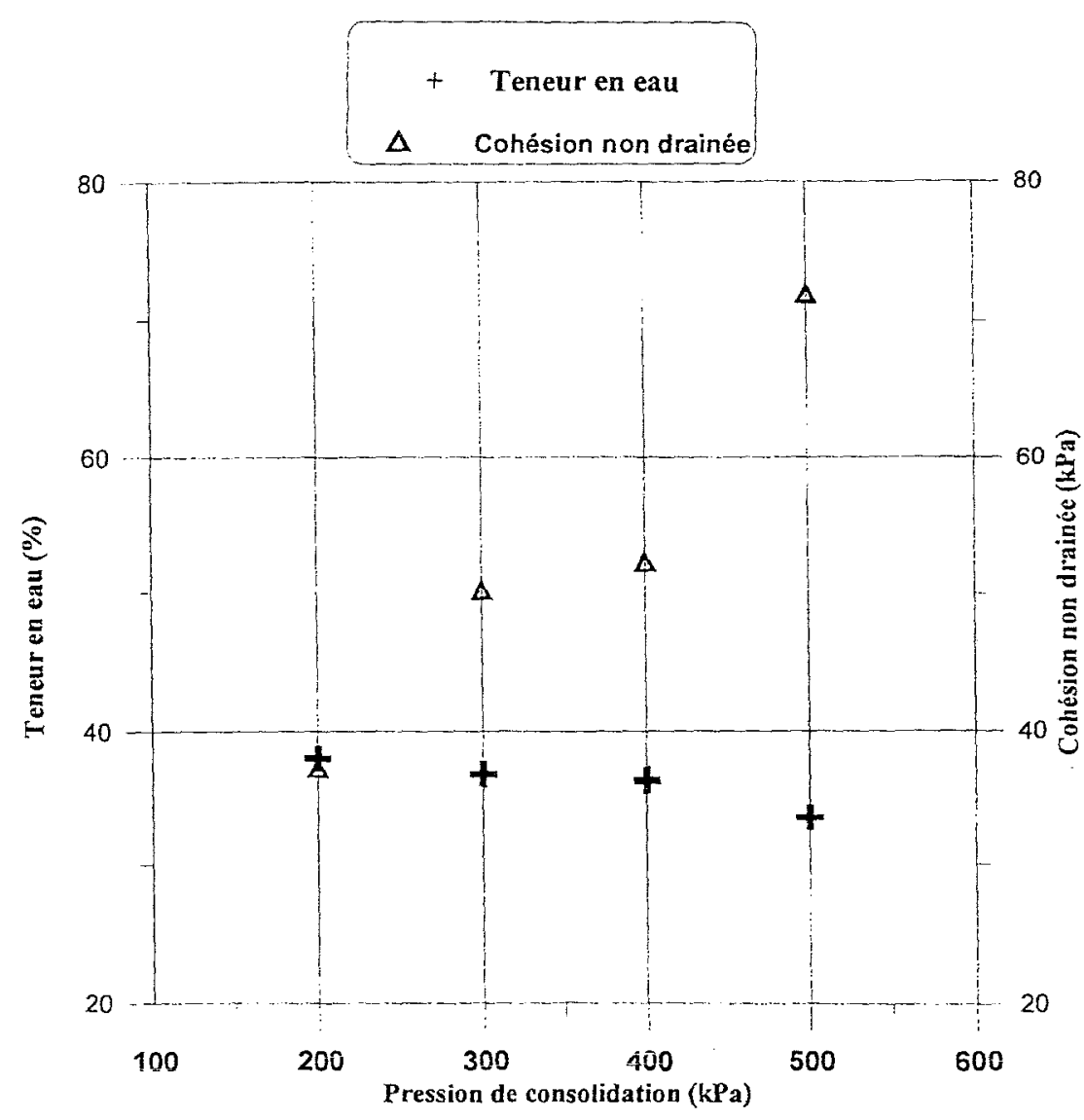

Figure 4: Variation de la teneur en eau et de la cohésion non drainée avec la pression de consolidation.

\section{Conclusion}

Les résultats obtenus lors de ces essais de reconstitution d'une argile ont montré que d'une part il est possible d'obtenir des échantillons homogènes, et que d'autre part l'opération de reconstitution est reproductible. Cette dernière est parfaitement maîtrisable lorsque la consolidation est effectuée avec soin par des pressions contrôlées en utilisant un vérin. Le contrôle de la consolidation par des capteurs de pression interstitielle placés dans le sol est souhaitable quand les dimensions de l'éprouvette le permettent. La procédure de consolidation décrite ici permet de moduler la pression finale sur l'échantillon en fonction des caractéristiques physiques et mécaniques que l'on veut obtenir pour l'échantillon reconstitué. Les résultats sont d'autant plus homogènes que les dimensions des éprouvettes sont petites. On peut également obtenir un gradient de cohésion, même faible, pour simuler les conditions réelles d'un sol dans son milieu naturel. L'étude de l'influence des paramètres d'essais sur les caractéristiques mesurées 
d'un sol peut donc être menée avec des échantillons reconstitués au laboratoire. Cette démarche présente un atout à l'expérimentateur qui dispose alors d'un sol de caractéristiques physiques et mécaniques homogénes et non affectés par le remaniement.

\section{Références bibliographiques}

Benamar A.,1992, Etude expérimentale du frottement latéral argile - pieu en cours de battage, Thèse de Doctorat, Université de Nantes - Ecole Centrale de Nantes, Juillet 1992, 169 pages.

Bat A., 1996, Problèmes de reconstitution d'éprouvettes de sols argileux en laboratoire, Actes des IVèmes Journées nationales Génie civil - Génie côtier, Dinard, 17-19 Avril 1996, pp. 479 - 487.

Craig R.F., Soil mechanics, Fifth edition, Chapman \& Hall, New York, 388 p. 